\title{
Téoros
}

Revue de recherche en tourisme

\section{Regard chinois sur le " plus beau paysage sous le ciel »}

\section{Benjamin Taunay}

Volume 29, numéro 2, 2010

Géotourisme

URI : https://id.erudit.org/iderudit/1024868ar

DOI : https://doi.org/10.7202/1024868ar

Aller au sommaire du numéro

Éditeur(s)

Université du Québec à Montréal

ISSN

0712-8657 (imprimé)

1923-2705 (numérique)

Découvrir la revue

Citer cet article

Taunay, B. (2010). Regard chinois sur le " plus beau paysage sous le ciel ». Téoros, 29(2), 26-34. https://doi.org/10.7202/1024868ar

\section{Résumé de l'article}

Cet article s'intéresse aux pratiques des touristes intérieurs en Chine en analysant le regard qu'ils portent sur la nature dans différents sites naturels du sud-ouest de la Chine, comme le fleuve Li, les grottes de Guilin ou d'autres sites de la région du Guangxi (voire du Guizhou). Une analyse qualitative des pratiques cherche à déterminer si, chez les touristes intérieurs, une " autre » manière de regarder la nature et les paysages, singulière et originale par rapport à celles connues en Occident, est avérée. d'utilisation que vous pouvez consulter en ligne.

https://apropos.erudit.org/fr/usagers/politique-dutilisation/ 


\title{
Regard chinois sur le "plus beau paysage sous le ciel»
}

\author{
Benjamin TAUNAY \\ Docteur en géographie \\ Postdoctorant à l'Université d'Angers, faculté d'ingénierie du tourisme (ITBS) \\ benjamin.taunay@gmail.com
}

\begin{abstract}
RÉSUMÉ: Cet article s'intéresse aux pratiques des touristes intérieurs en Chine en analysant le regard qu'ils portent sur la nature dans différents sites naturels du sud-ouest de la Chine, comme le fleuve Li, les grottes de Guilin ou d'autres sites de la région du Guangxi (voire du Guizhou). Une analyse qualitative des pratiques cherche à déterminer si, chez les touristes intérieurs, une «autre» manière de regarder la nature et les paysages, singulière et originale par rapport à celles connues en Occident, est avérée.
\end{abstract}

Mots-clés: Chine, tourisme intérieur chinois, paysage, nature, Guilin.

En Chine, la politique d'ouverture et de réforme, initiée à partir de 1975 et appliquée dès 1978, a conduit à l'apparition d'une classe moyenne chinoise et à l'augmentation progressive des flux de touristes intérieurs chinois dans la plupart des sites et lieux touristiques du pays. En particulier dans les années 1990, le tourisme intérieur chinois a progressé très rapidement. De 1992 à 1995, le nombre de touristes intérieurs a augmenté de plus de $20 \%$ chaque année. C'est pour encourager le développement de ce tourisme que le gouvernement a réduit progressivement la durée du travail hebdomadaire et a attribué des congés payés. Le $1^{\text {er }}$ mai 1995, la semaine de travail est passée de 44 à 40 heures. L'année 1996 est ensuite déclarée «année des loisirs et des vacances", et en 1997 sont instaurés les fins de semaine non travaillées pour tous les salariés. Surtout, le 18 septembre 1999 a vu l'instauration de trois jours de congé payé par an : la fête du printemps, le fête du travail ( $1^{\text {er }}$ mai) et la fête nationale ( $1^{\text {er }}$ octobre). «[A]vec les week-ends qui encadrent chacune de ces trois fêtes, les salariés disposent ainsi de trois «longs congés» (changjia) de sept jours. Ce sont les «semaines en or» (huangjin zhou $)^{1} »$ (Thireau, cité par Sanjuan, $\left.2006: 126\right)$. Ces trois semaines ont depuis été réduites à deux en novembre 2007 : la semaine d'or autour du $1^{\mathrm{er}}$ mai (anciennement la plus longue) a été supprimée, ne laissant de fériée que la seule journée du $1^{\mathrm{er}}$ mai. Les trois autres jours fériés ont été redistribués sur des fêtes traditionnelles chinoises (un jour à la fête des Morts située entre le 4 et le 6 avril, un à la fête des Bateaux-Dragons qui est le cinquième jour du cinquième mois lunaire, soit environ en juin, et un troisième à la fête de la Mi-automne le quinzième jour du huitième mois lunaire), et le nombre total de jours de congé par an est passé de 10 à 11.

Les touristes intérieurs chinois sont aujourd'hui environ 300 millions (Taunay, 2009). Pourtant, il existe très peu de documentation sur les caractéristiques de ces touristes. Tim Oakes (1998) a insisté sur leur recherche de modernité, Pal Nyíri (2006 et 2008) et Béatrice David (2007) nous ont montré le poids de l'État dans la définition, la stimulation et le contrôle des pratiques touristiques chinoises. Nous ne savons en revanche presque rien des pratiques touristiques chinoises face à la nature, alors même que la Chine est la première civilisation paysagère (Berque, 1995). Peut-on dès lors supposer que les pratiques des touristes chinois sont les mêmes que celles connues ailleurs dans le monde? Dans quelle mesure, les touristes chinois associent-ils des significations particulières aux formes naturelles des géosites?

L'hypothèse que la nature du paysage observé est probablement différente entre Occidentaux et Chinois se justifie tout d'abord parce que le terme de paysage recouvre de multiples significations en chinois mandarin. Ensuite, les touristes chinois du continent se dirigent d'abord vers les grands centres urbains de l'Est dans une quête de modernité (Nyíri, 2006) et apprécient ensuite des lieux de l'intérieur du pays, des «sites pittoresques célèbres» (mingsheng ${ }^{2}$ ) connus depuis l'Antiquité et visités par des nobles lettrés, sortes de parcs nationaux aménagés en zones 
ILLUSTRATION 1 : Guilin, au nord-est de la région autonome Zhuang du Guangxi (compilation de l'auteur; source : Wang, 2004)

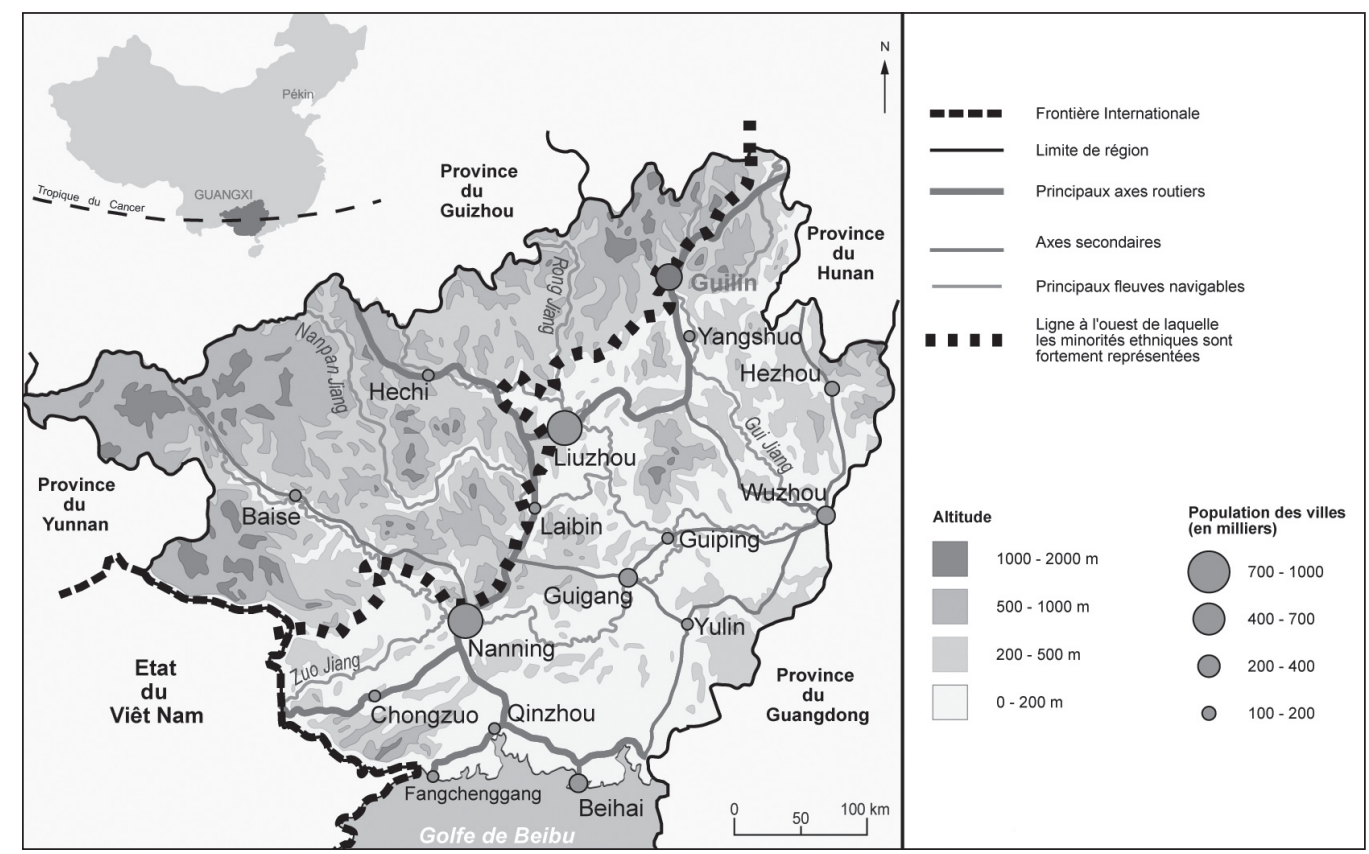

d'intérêt paysager et historique d'importance nationale (zhongdian fengjing mingshengqu $u^{3}$ ) par l'État communiste entre 1978 et 2005. Le «regard touristique» («tourist gaze», Urry, 1990) chinois propose-t-il donc une nouvelle métaphore des rapports sociaux et des rapports à l'espace, différente de ce qui est déjà connu en Occident où est né le tourisme? Une importante partie des pratiques touristiques consiste en effet à « regarder des paysages différents de ceux du quotidien » (Stock, 2005), et l'on sait que la réalité chinoise contemporaine est profondément différente de ce qui se passe ailleurs dans le monde : en plus de reposer sur la plus ancienne civilisation au monde, la société chinoise est en quête de modernité et est prise dans des "mutations accélérées » (Sanjuan, 2007). Quel est donc le regard chinois sur le géotourisme? Est-il également fondé, comme le définit la National Geographic Society (NGS), sur un tourisme qui «soutient et met en valeur une destination selon son caractère géographique, son environnement, sa culture, son esthétisme, son patrimoine et le bien être de ses habitants» (NGS, 2009: 1)?

Pour avancer des éléments de réponse à cette question de la nature des pratiques spatiales et des modalités de contemplation des géosites (un espace qui permet d'observer des éléments et des phénomènes géologiques intéressants pour la compréhension de la géologie) des Chinois, cet article s'intéressera au cas particulier des paysages de Guilin, dans la province méridionale du Guangxi (voir illustration 1). Ces paysages font partie de ceux que tout un chacun, en Chine, connaît et convoite de visiter au moins une fois dans sa vie, en tant que quintessence du paysage défini comme celui formé de montagne et d'eau qui court (shanshui ${ }^{4}$ ). Cette esthétique chinoise (Hu-Sterk, 2004) sera ici analysée grâce à des observations de terrain menées dans différents géosites à Guilin entre 2005 et 2008 et tentera de comprendre si, derrière des différences de regards et de pratiques touristiques, il y a une différence de définition du géotourisme et des géosites. Pour la première partie de l'article, les observations ont été réalisées lors de trois croisières sur des bateaux fréquentés exclusivement par des touristes intérieurs chinois et lors d'entretiens avec ces touristes. Quant à la deuxième partie de l'article, elle se fonde principalement sur des observations couplées à des recherches documentaires dans différents géosites du Guangxi et du Guizhou.

\section{Des pratiques spatiales différentes dans la "galerie de peintures» de Guilin}

De toutes les visites dans la région de Guilin, la croisière sur le fleuve Li est considérée incontournable pour les touristes intérieurs comme pour les internationaux. Pourtant, il semble que chaque population touristique n'observe pas les mêmes éléments lors de la croisière.

\section{Guilin, la quintessence du paysage à la chinoise}

Guilin, une ville de 700000 habitants, localisée au nord-est de la province du Guangxi, jouit d'une aura très importante dans tout le pays. Cette province est particulière en ce sens que c'est une des cinq régions dites «autonomes» de Chine. Ceci est attribuable au grand nombre de minorités ethniques présentes dans la province. En Chine, la population han constitue près de $96 \%$ de la population, localisée principalement dans les plaines, laissant les marges montagnardes aux minorités. Dans le cas du Guangxi, la principale minorité est celle de Zhuang, d'où le nom de la région. Ville apparue il y a plus de 2000 ans, Guilin a longtemps été la capitale de la province du Guangxi avant que Nanning ne lui ravisse ce titre à la fin des années 1950 avec l'instauration de la région autonome de Zhuang du Guangxi. Les touristes, Chinois et étrangers, qui se rendent au Guangxi visitent cependant d'abord Guilin. Ils sont motivés par ses paysages qui se trouvent dans l'espace urbain et surtout au bord du fleuve Li (lijiang ${ }^{5}$ ), qui mène de la ville de Guilin au village de Yangshuo $^{6}$, à $83 \mathrm{~km}$ au sud de Guilin. Une expression populaire 
dit notamment que les plus beaux paysages de Chine sont à Guilin, et que les plus beaux paysages de Guilin sont à Yangshuo ( "guilin shanshui jia tian xia, yangshuo shanshui jia guilin ${ }^{7}$ »). C'est pourquoi la croisière sur le fleuve Li, entre Guilin et Yangshuo, représente une visite «incontournable»: plus de 10 millions de touristes intérieurs chinois ont visité la région de Guilin en 2007 pour près d'un million de touristes étrangers.

L'important nombre de touristes intérieurs est la conséquence de ce que la société chinoise, depuis plus de 2000 ans, porte un regard «obsessionnel» sur les phénomènes naturels et les retranscrivent dans les arts : première civilisation paysagère, la Chine a en effet été la première à proposer l'usage d'un ou plusieurs mots pour dire "paysage», une littérature (orale ou écrite) décrivant des paysages ou chantant leur beauté, des représentations picturales de paysages, des représentations jardinières traduisant une appréciation proprement esthétique de la nature; il ne s'agit donc aucunement de jardin de subsistance (Berque, 1995). La nature n'est alors pas figée. Elle est au contraire en mouvement : c'est un «souffle» qui donne vie à l'espace, que les lettrés ont depuis toujours essayé de représenter dans leur peinture de paysage. "[L]e tableau peint doit donc être envisagé comme un corps vivant; c'est ainsi que pour un paysage donné on parle d'ossatures (rochers), d'artères (cours d'eau), de muscles (arbres), de respiration (nuages), etc.» (Cheng, 1989 : 143). La quintessence de ce type de paysage ${ }^{8}$, se trouve à Guilin : la petite forêt d'osmanthus, arbres qui produisent des fleurs blanches très odoriférantes semblables au jasmin. Les preuves de cette quintessence sont les anciens poèmes chantant la beauté «naturelle» de la ville, gravés dans les pierres, à la base des nombreuses collines de la ville, notamment la colline de la trompe de l'éléphant, la colline Fubo et la colline des couleurs accumulées (die cai ${ }^{9}$ ), toutes trois dans l'ancien cœur historique de la ville.

La calligraphie indique que «le paysage de Guilin est le plus beau sous le ciel». Ce tableau est peint à la manière des anciennes toiles produites pendant l'époque impériale, où l'important est de jouer sur la «fadeur» (Jullien, 1993) des couleurs. Pas de tons vifs, mais plutôt une tendance à utiliser des couleurs pâles permet de saisir une «respiration» du site (voir l'introduction générale). Ici, les collines se reflètent dans le fleuve Li, alors que la brume entoure les sommets des pics. L'artiste, Li Bing, peint des paysages à la manière des peintres de l'époque classique.

\section{Ce que voient les touristes dans le fleuve $\mathrm{Li}$}

Guilin est le point de départ de la croisière sur le fleuve Li dont les paysages ont construit la renommée de la région : l'expression précédente "le plus beau paysage est à Guilin» a été inspirée par ses pics karstiques qui tombent dans le fleuve et s'y reflètent. Nombreux sont les peintres qui furent inspirés par les paysages entre Guilin et Yangshuo, à tel point que cette partie du fleuve est aujourd'hui considérée comme une "galerie de peintures» (hualang $\left.{ }^{10}\right)$. Les poètes qui ont décrit le paysage du fleuve Li sont également innombrables. Han Yu par exemple, un poète célèbre sous la dynastie des Tang (608-907 apr. J.-C.), abondamment cité dans les guides imprimés et sur internet, a vivement chanté la beauté de ce lieu et une de ses phrases est restée célèbre : «[L]e fleuve Li forme une ceinture de gaze verte, les collines sont comme des épingles à cheveux en jade bleu.»
Les touristes intérieurs associent donc aujourd'hui ce lieu à de nombreuses peintures de paysage, et c'est pourquoi cette croisière est tant briguée par tous ceux qui se rendent à Guilin pour la première fois.

Les touristes internationaux qui se rendent à Guilin sont eux aussi extrêmement désireux d'effectuer cette croisière, mais leurs motivations, ainsi que les thèmes sur lesquels se pose leur regard, divergent sensiblement. D'ailleurs, ces deux populations touristiques sont séparées sur des bateaux différents (Le prix n'est également pas le même : environ 300 yuans pour les touristes chinois et 400 yuans pour les internationaux.). Une enquête auprès des internationaux mettra en exergue leur préférence pour un temps ensoleillé (voir illustration 2), temps qui convient mieux aux photos de panorama. Les Occidentaux voient d'abord dans les paysages du fleuve Li des formes karstiques, ou formations géologiques couvertes de végétations. Pour ces touristes, les pics sont en apparence noirs, comme une «forêt de pierres ceinturant le fleuve». Ensuite, ils s'intéressent aux villages de pêcheurs qui bordent le fleuve ainsi qu'à la vie quotidienne de leurs habitants. Ceci montre l'écart remarquable entre le regard occidental et chinois : ce dernier porte peu d'attention à la vie des populations locales, et la dimension «sciences de la terre» n'est que très peu abordée dans les discours destinés à ces touristes. En revanche, elle prend une place beaucoup plus importante chez les internationaux. Il semble donc que l'intérêt pour le géotourisme va de pair avec la découverte de l'autre pour les internationaux là où il est associé à une connaissance de la culture (ici les peintures et les descriptions des artistes) chez les touristes chinois.

L'illustration 2 nous montre un décor qu'apprécient les touristes occidentaux. Le temps est ensoleillé, faisant ressortir les couleurs des collines qui entourent le fleuve Li. Le vert de la végétation tranche bien avec le gris-marron des collines, ainsi qu'avec le bleu clair du ciel. Une importance est également donnée à la vie locale, manifestée ici par l'élevage de bovins au bord du fleuve et parfois par les villages qui apparaissent au détour des méandres du fleuve.

Notons maintenant le regard porté sur les paysages du fleuve Li par les Chinois : en premier lieu, ils n'ont rien contre un temps pluvieux ou nuageux, voire même brumeux. Ce temps n'est pourtant pas maussade pour les touristes chinois. Un temps couvert apporte plus de nuances aux collines et fait ressembler le décor aux peintures anciennes évoquées plus haut (voir illustration 3). Les nuages et la brume font ainsi changer le paysage de minute en minute, faisant apparaître ou disparaître périodiquement des parties de cette peinture-paysage. Les formes qui naissent et meurent sont alors sujets de longues discussions sur le bateau. Le regard des touristes chinois ne se laisse pas capter par les formes géologiques, mais par des représentations standardisées du passé, connues et facilement identifiables par tous. Pour cette raison, tous se prennent en photo au même endroit, dans des poses stéréotypées (infra). Enfin, les touristes intérieurs s'intéressent peu aux populations locales : les maisons des pêcheurs sont au mieux des éléments d'un tableau, et ses habitants sont souvent même considérés comme des populations arriérées. Comme souligné en introduction, ce rapport à la modernité est fondamental dans les pratiques touristiques chinoises en Chine. Les espaces ruraux sont perçus comme 


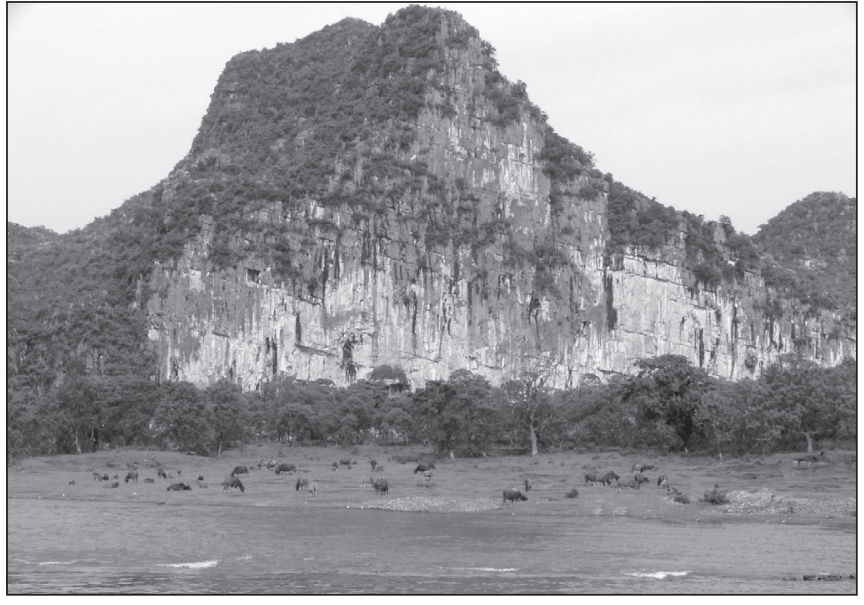

ILLUSTRATION 2 : Un paysage du fleuve Li, apprécié par les touristes occidentaux (photo : Benjamin Taunay).

des espaces «répulsifs», réservés à la population paysanne de laquelle les touristes, en majeure partie originaires des villes, essayent au maximum de se distinguer.

Ces premiers éléments croisés permettent donc d'entrevoir la différence qui caractérise le regard des touristes internationaux et des touristes intérieurs sur la nature en général, et sur le paysage en particulier. Comme l'a indiqué l'anthropologue Pal Nyíri dans ses travaux (2006 et 2008), cette différence est pour une large part le résultat d'une forte impulsion de l'État. Ainsi, certains «sites pittoresques célèbres» (Mingsheng ${ }^{11}$ ), célébrés par les artistes depuis l'Antiquité et par les lettrés depuis la dynastie des Ming (notamment le $15^{\mathrm{e}}$ siècle), ont été revalorisés par l'État chinois dès la fin des années 1970, dans un projet de construction nationale au travers du tourisme. Dans le but de constituer un ensemble de pratiques touristiques, l'État a également publié de nombreuses compilations de récits de voyages classiques (youji), alors renommés «littérature touristique» $(\text { lïyou wenxue })^{12}$.

\section{Les éléments regardés lors de la visite}

Cette impulsion de l'État se retrouve également dans une autre modalité du regard porté sur les paysages du fleuve Li. En effet, les touristes intérieurs scrutent attentivement les collines et leurs formes : les visiteurs de l'époque classique avaient largement décrit ce que leur inspiraient les formes des collines le long du fleuve en le consignant dans des carnets de voyage. Les croisières se font donc aujourd'hui toujours au rythme de la découverte des différentes formes associées aux collines et on constate ainsi une forte importance des géosites dans l'approche paysagère chinoise. Les discours des guides touristiques, prononcés par de puissants haut-parleurs pendant la visite, n'arrêtent pas de le souligner : ce sont les géosites, admirés et décrits par les illustres personnages du passé, que les touristes doivent à leur tour regarder dans une sorte de continuum culturel et temporel. Les collines des «coqs de combat» sont, par exemple:

Two hills in shape of two fighting roosters face each over across river. The hill on the east bank is Chuanshan Hill and resembles a rooster opening its eyes wide and holding

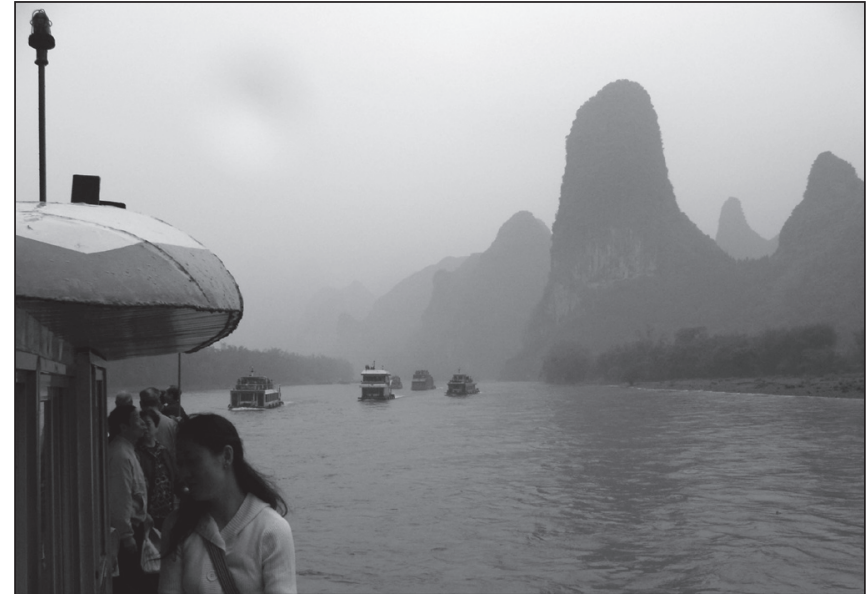

ILLUSTRATION 3 : Paysage brumeux du fleuve Li, apprécié par les touristes intérieurs (photo : Benjamin Taunay).

up its long tail. While the west one looks like a rooster with its crest lifted and is named Gamecock Hill [...] Looking back from there, the fighting gesture of these two roosters jumps directly into your eyes [...] Vivid and lifelike, it is listed as one of the ten marvellous spectacles of the Li River (discours d'un guide chinois anglais).

Ce commentaire, issu d'un guide en anglais, se retrouve dans d'autres guides, dans différentes langues, ce qui montre le caractère stéréotypé des commentaires des guides. Ce point renforce ainsi l'analyse de Nyíri sur le rôle de l'État et qui montre le poids de cet acteur dans le contrôle des discours sur et dans les sites touristiques.

Ou bien encore la colline des «huit immortels traversant le fleuve» est ainsi décrite :

[Ces] huit crêtes [sont] taillées comme des sculptures naturelles. Ce sont les huit immortels. Un jour, à l'issue d'un de leurs voyages, ils arrivèrent au fleuve Li. Trouvant l'endroit magnifique, ils décidèrent d'y rester, mais ce faisant ils acceptèrent de perdre leur immortalité et, plutôt que de retourner au royaume des cieux, ils préferent être transformés en pierres pour continuer à admirer le spectacle du lieu (discours d'un guide chinois francophone).

Ces exemples ne sont pourtant pas isolés, car nombreuses sont les montagnes qui ont été nommées (voir tableau 1).

Ces 27 collines sont les principales attractions de la croisière sur le fleuve Li. D'une certaine manière, ces descriptions ressemblent un peu à celles des sites naturels en France à la fin du $19^{\mathrm{e}}$ et au début du $20^{\mathrm{e}}$ siècle : l'intérêt à cette époque n'était-il pas d'observer les formes des grottes, des cheminées de fée, des roches multiformes, etc.? Dans les deux cas, il faut peut-être voir là une volonté de construction nationale par l'État (pour le cas français, voir ainsi Thiesse, 1999). Au fur et à mesure de la visite, un haut-parleur décrit les formes associées à ces différentes collines, et les touristes basculent périodiquement de droite à gauche du bateau selon le côté où se situe la colline. Tous les touristes écoutent les descriptions faites par le guide et, une fois la description terminée, tous se pressent et font la queue pour 


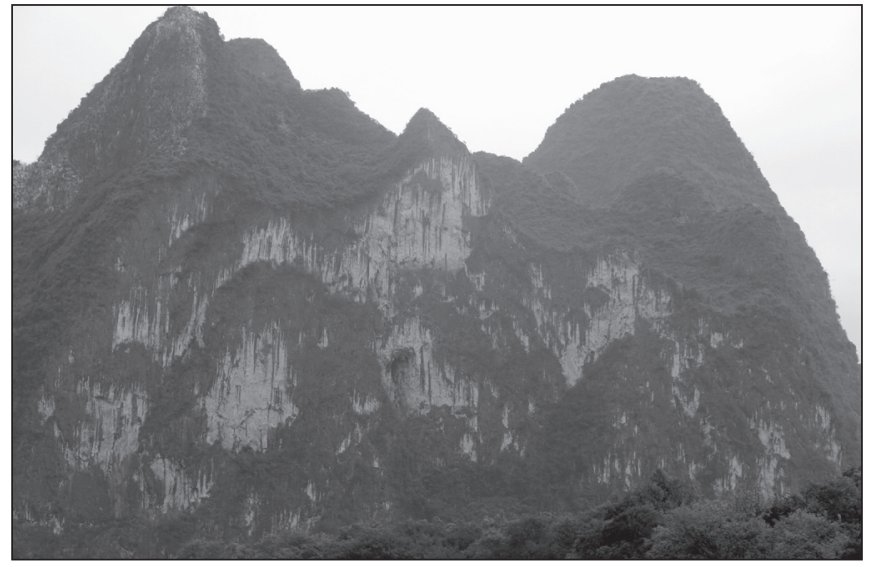

ILLUSTRATION 4 : La colline de la fresque des neuf chevaux (photo : Benjamin Taunay).

Tableau 1: Les collines nommées le long du fleuve Li entre Guilin et Yangshuo

\begin{tabular}{l|l}
\hline 1. Les étranges cimes de la forêt & $\begin{array}{l}\text { 15. Le lion qui gravit la colline } \\
\text { des cinq doigts }\end{array}$ \\
\hline 2. La grenouille qui se réveille & 16. La cage du poulet \\
\hline 3. La meule & 17. La tortue qui grimpe sur la colline \\
\hline 4. La falaise de la gorge des bœufs & 18. Huit immortels traversant le fleuve \\
\hline $\begin{array}{l}\text { 5. Les neufs dragons jouant } \\
\text { au dessus de l'eau }\end{array}$ & 19. La queue du poisson \\
\hline 6. Un heureux mariage & 20. La fresque des neuf chevaux \\
\hline 7. La grenouille qui traverse la rivière & 21. Le lion observant les neuf chevaux \\
\hline $\begin{array}{l}\text { 8. La femme qui attend } \\
\text { le retour de son mari }\end{array}$ & 22. Le gant \\
\hline 9. Le bac d'un côté & 23. La magnifique jeune fille \\
\hline $\begin{array}{l}\text { 10. La broderie } \\
\text { 11. Le meunier au travail }\end{array}$ & 24. Le chameau traversant le fleuve \\
\hline 12. La vague de pierre & 25. L'escargot \\
\hline 13. Le porte-stylo & 26. La carpe \\
\hline $\begin{array}{l}\text { 14. Le grand-père qui regarde } \\
\text { la pomme }\end{array}$ & 27. La tête du dragon \\
\hline Source : Bureau du tourisme de Guilin, 2008. &
\end{tabular}

se faire prendre en photo devant ladite scène (les descriptions associent toujours la forme de la colline à une histoire ou à une légende). Rares sont les touristes qui n'écoutent pas les informations données par le guide et, de manière générale, tout ce qui est nommé (ce sont toujours des phénomènes naturels, comme les gorges, xia, par exemple) est photographié.

Ce cliché a été pris non depuis le fleuve, mais depuis sa rive droite. Il permet de souligner l'importance de cette colline, eu égard à la taille des maisons (au premier plan). La colline des neuf chevaux est la plus connue de toutes celles qui jalonnent le parcours de Guilin à Yangshuo. Elle est même considérée comme une peinture, c'est-à-dire une œuvre d'art.
La réponse des touristes aux explications du guide est à peu près la même pour tous. Les touristes chinois sont admiratifs devant le «plus beau paysage sous le ciel» et ils n'hésitent pas à le manifester par des exclamations à chaque fois qu'ils découvrent une nouvelle colline, et donc une nouvelle scène. Dans l'exemple de la colline de la fresque des neuf chevaux, une des plus connues (voir illustration 4), les touristes doivent parvenir à distinguer neuf chevaux. Le guide explique ensuite que celui qui arrive à tous les voir est un génie, un individu digne d'être classé premier à l'ancien concours impérial :

[L]a légende raconte que ces neuf chevaux, effrayés par un peintre, en tentant de s'enfuir se seraient retrouvés bloqués dans la pierre, faisant de cette colline une véritable fresque. Il est difficile de distinguer tous les chevaux et on dit que celui qui pouvait identifier chacun des neuf chevaux serait le disciple numéro un, c'est-à-dire le titre conféré à celui qui a obtenu le premier prix à l'examen impérial le plus élevé (guide chinois francophone).

Tout le monde se presse donc sur le pont pour observer et essayer de voir, déclarant en avoir vu au moins un certain nombre. Les discours vont bon train sur celui dont le regard en a capté le plus. Les pratiques sont alors clairement collectives et assez uniformes. Il est possible de rapprocher ce phénomène d'un certain patriotisme, voire d'un nationalisme. Les caractéristiques des pratiques touristiques ressemblent notamment à celles observées par Ku Ming-Chun (2006), dans l'ouest de la Chine : «[F]or example, a domestic tourist may broach the topic of how foreigners stole the trasure of the Mogao Caves, and immediately the other tourists would add their own comments to this topic... The staff guide had no way to stop the tour group, which turned into something like a forum of patriotism and nationalism» (Ming-Chun, 2006, cité dans Nyíri, 2008 : 166).

Les pratiques touristiques évoluent au cours des quatre heures que dure la croisière. La première heure se passe sur le pont, selon le descriptif que nous venons de préciser (vers $9 \mathrm{~h}$ ou $10 \mathrm{~h}$ ) : les touristes y prennent beaucoup de photos au rythme des descriptions du guide. Les touristes intérieurs sont clairement attentifs aux collines karstiques et aux explications du guide qui, grâce au haut-parleur placé sur le toit du bateau, allie la description des formes des collines aux légendes qui leur sont associées. Selon l'importance donnée à telle ou telle colline, une file de touristes se constitue alors pour être pris en photo devant le pic. Ensuite, pendant la deuxième heure, les touristes commencent à regarder davantage les paysages, à être plus attentifs à la nature et moins attentifs au guide (vers $10 \mathrm{~h}$ ou $11 \mathrm{~h}$ ). Le tournant de la visite intervient alors au moment du repas de midi, servi entre $10 \mathrm{~h} 50$ et $11 \mathrm{~h} 05$ : plus de la moitié des touristes préfere rester dans le bateau plutôt que de remonter sur le pont une fois le repas pris. Singulièrement, peu ressortent pour prendre en photo les environs du village de Xingping, site qui a été reproduit sur le billet de 20 yuans. Ceux qui remontent le font en général pour peu de temps, la plus «belle» partie de la croisière étant selon eux passée. On observe d'ailleurs depuis 2009 une recrudescence du nombre de paysans qui proposent une croisière réduite, d'environ deux heures, centrée sur la partie du fleuve considérée comme la plus belle. Cette «nouvelle» forme de croisière se fait sur des bateaux beaucoup plus petits (entre quatre et dix places) (Cette question de la participation des communautés locales est 
à approfondir, à partir de l'exemple suivant : Wang, Yang, Chen, Yang et Li, 2010). Cet aspect suggère l'idée que les touristes intérieurs montrent également de l'importance aux commodités matérielles, même dans des pratiques touristiques de découverte. C'est pourquoi la plupart des séjours touristiques se font dans ou à proximité d'un important centre urbain.

\section{Les grottes et leurs formes mises en valeur (exemples à Guilin et au Guizhou)}

Pendant la croisière sur le fleuve $\mathrm{Li}$, de nombreuses grottes sises au bord du fleuve sont signalées, nommées, en tant que parties intégrantes des paysages. À ce titre, il est intéressant d'observer les pratiques qui y ont cours. Comme les collines du fleuve $\mathrm{Li}$, de nombreuses formes dans les cavités sont nommées, en plus d'être mises en valeur par un jeu d'éclairage.

\section{Les formes des grottes sont également nommées}

En plus des nombreuses collines karstiques ouvertes au tourisme dans la région de Guilin, de nombreuses grottes sont également aujourd'hui ouvertes au public. Là aussi le regard des touristes internationaux et des touristes intérieurs diffère sur de nombreux points. Les touristes intérieurs n'accordent que peu de valeur à l'origine géologique et à la formation des stalagmites et stalactites. Le discours du guide n'y consacre en effet que quelques phrases au début de ses explications (d'ailleurs souvent à l'entrée de la grotte). Le géotourisme en Chine pour les Chinois ne se centre donc pas sur les mêmes aspects qu'ailleurs dans le monde, notamment celui occidental (Il faudrait donc peut-être revoir la pertinence du concept de géotourisme à la lumière de l'expérience chinoise, c'est ce que suggère la forme qui l'emporte sur la construction de celle-ci.).

Comme pour les collines du fleuve Li, le point de mire n'est pas l'origine des formations calcaires, mais les formes qu'elles revêtent. L'observation d'une stalactite est pertinente car elle représente un lion, une fleur de lotus, etc. (voir illustration 5). Comme au fil de la croisière, à chaque forme est associée une histoire qui fait appel à l'imagination des touristes. Par exemple, dans la grotte de la flûte du roseau se trouve un endroit nommé «le soleil du matin au dessus de la forêt des lions». Ceci parce qu' «il semble ici y avoir une forêt vierge. À l'intérieur, vous voyez avec sa tête, le corps et la queue. Il y a aussi des lionceaux, au pied de cette montagne. Chaque matin, quand le soleil se lève, ce groupe de lions viendra et jouera dans cette forêt» (traduction libre d'un guide chinois). Les exemples, circonstanciés, different d'une grotte à l'autre. Une liste exhaustive n'est donc pas possible, mais le schéma, basé sur l'analogie, est toujours le même.

L'illustration $5 \mathrm{du}$ "lion rugissant» est intéressante. Cette "scène» est un des endroits clés de la grotte, un des points où le guide reste le plus longtemps, en partie à cause des stands de photographes qui s'y trouvent. Dans l'exemple de la grotte de Yiling, le visiteur peut dès l'entrée voir les points les plus importants : le village de montagne pendant la nuit, la forêt dense, la neige dans le nord de la Chine, etc. Ce sont d'ailleurs ces points qui seront le plus "admirés» et les stands de prise en photo se trouvent près des plus «beaux» d'entre eux. Les noms des sites sont enfin intéressants, ils nous apprennent beaucoup sur l'esthétique chinoise, fondée ici sur des références à des espaces naturels.

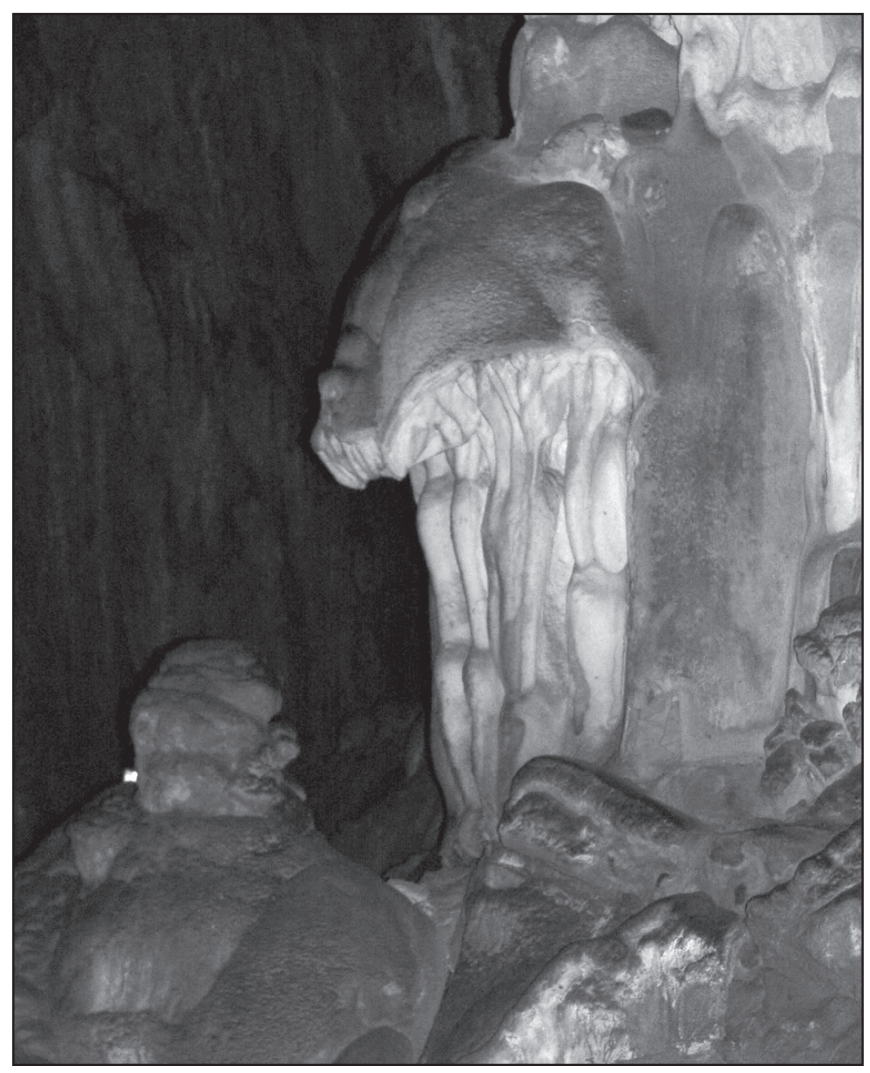

ILLUSTRATION 5 : Grotte de Zhijin, province du Guizhou, «lion rugissant » (photo : Benjamin Taunay).

Parfois le guide demande même aux touristes de prêter l'oreille pour entendre un certain son : "Au dessus, lorsque nous tournons la tête un peu sur la gauche, il y a une grande cascade descendant de la falaise montagneuse. Il semble que nous pouvons entendre le son de la chute d'eau». Cet exemple est intéressant, car il montre que les formes données aux formations géologiques ne sont pas attribuées au hasard, mais qu'elles correspondent à des visions anciennes de voyageurs du passé. «Et cela nous fait rappeler un poème très connu écrit par Li Bai, un poète de la dynastie Tang : «[L]a cascade se déversant de trois mille pieds de haut, c'est comme la voie lactée tombant du ciel.» » Ici encore, les formes regardées aujourd'hui sont celles qui l'étaient hier : les visiteurs empruntent encore les circuits ébauchés par les visiteurs du passé. Dans le Guangxi par exemple, le géographe Xu Xiake (1587-1641) a ainsi visité la grotte de Yiling, ainsi que la grotte de la flûte du roseau située à Guilin (On peut retrouver en langue française une partie du récit du voyage de Xu Xiake : Dars, 1993.).

Dans de nombreuses grottes pourtant, l'attribution de noms n'a été réalisée qu'assez récemment, c'est le cas de la «statue de la liberté» sise dans la grotte de la flûte du roseau. Ce nom ne provient bien évidemment pas de l'époque classique et de ses visiteurs lettrés, mais de certains touristes qui ont remarqué des formes jusqu'alors passées "inaperçues». Le guide a donc pris note de cette nouvelle forme avant de l'intégrer dans son discours. Le guide encourage même les touristes à stimuler leurs 
capacités imaginatives : «J'espère vraiment qu’ici, vous pourrez utiliser votre imagination lors du parcours.» C'est pourquoi dans les visites des grottes, les noms anciens correspondant à la culture chinoise («la gorge de la fleur du soleil», «le roi singe du voyage vers l'ouest», "un dragon bleu s'enroulant autour de la pagode», etc.) côtoient aujourd'hui des noms plus récents ( la statue de la liberté», «les gratte-ciels de Shanghai», «les chutes du Niagara», etc.). On aura noté la participation active de Chinois friands d'analogies.

Les touristes admirent largement les formes données aux formations géologiques et, là encore, poussent souvent des exclamations à la découverte de nouvelles formes. Également très attentifs aux explications du guide, ils déplacent leur regard au rythme de ses explications, à de rares exceptions près. Un schéma des touristes dans une grotte est donc assez simple à réaliser : ceux-ci forment des points (agglomérations de touristes) autour des attractions et, entre ces points, ce sont des lignes vides : les touristes ne s'arrêtent que devant les sites nommés. Les groupes dans les grottes sont ainsi assez uniformes, les initiatives personnelles ou apartés plutôt rares.

\section{Les couleurs des grottes et la modernité}

Une autre étape confère de la «valeur» à un site : les visiteurs illustres de l'époque contemporaine. Nombreux sont les clichés photographiques de Deng Xiaoping, pris lors de sa visite de la grotte de la flûte du roseau, postés tout au long du parcours. Pour les touristes chinois, «gagner de la face» est une préoccupation constante. C'est donc là un encouragement évident à visiter cette grotte : si le petit timonier est venu observer les formes des cavités karstiques, alors c'est une affaire sérieuse que chaque touriste à Guilin doit également réaliser. La grotte est d'ailleurs maintenant nommée grotte "présidentielle». Dans une société où le collectif prime sur l'individu (au profit d'une cohabitation, d'une «harmonie»), une telle visite ne fait donc qu'amplifier le conformisme des pratiques touristiques. D'ailleurs, les exemples de visites de hauts dirigeants sont nombreux dans les différents sites et lieux touristiques du pays. À chaque fois, le but est de lancer le lieu et de montrer l'exemple.

Est-ce pour autant que les touristes ne portent pas d'attention aux couleurs éclatantes qui éclairent les différentes formes mises en valeur? En fait, les grottes de la région de Guilin, de la province du Guizhou, jusqu'à la municipalité de Nanning, sont toutes éclairées par des couleurs qui paraissent exubérantes à première vue. C'est en tout cas la première réaction des touristes étrangers, qui, pour les qualifier, utilisent allègrement l'épithète de «kitch». Pourtant, les touristes chinois ne s'étonnent guère de ces différentes couleurs, et semblent même les apprécier. Les touristes chinois interrogés trouvent que l'atmosphère sombre et peu accueillante se trouve de suite embellie : la grotte devient chaleureuse, voire magique. Là encore, le guide demande aux touristes d'utiliser leur imagination.

L'enthousiasme chinois pour les couleurs éclatantes trouve sans doute son origine dans ce que la civilisation chinoise associe à ces couleurs, la perception du spectre coloré n'étant pas la même que chez les populations occidentales. Pour preuve, chaque grotte se targue d'être la "plus belle sous le ciel» (tian xia di yi dong ${ }^{13}$ ), ce qui laisse envisager la «beauté» des espaces ainsi éclairés. De Guilin à la province du Guizhou, en passant par Nanning, toutes les publicités de grottes font référence à l'expression littéraire "le plus beau paysage est à Guilin». Comme dans l'exemple du fleuve $\mathrm{Li}$, certaines grottes usent de l'expression "galerie de peintures» (hualang), en référence aux effets que diffusent les lumières dans les cavités et sur les murs de celles-ci. Une publicité décrivant la grotte de Zhijin va jusqu'à prétendre qu'elle est le "palais des fées» (shenxian dong $\left.f u^{14}\right)$.

Les couleurs de la grotte Guan, située à environ 30 kilomètres au sud-est de la ville de Guilin, sont très vives. Elles contrastent fortement avec la recherche de «fadeur» constatée précédemment dans le cas de la croisière sur le fleuve Li. La totalité des sites clés dans les grottes est illuminée de cette manière dans les provinces du Guizhou et du Guangxi, mettant en valeur un «feu d'artifice», là un «lion rugissant», etc. Ce point s'explique par la recherche de modernité des touristes chinois. Ici les lumières rappellent celles des villes modernes et attirantes, ailleurs la commodité d'une chambre de luxe.

Une autre explication plausible a trait à la quête de modernité des touristes intérieurs visitant ces grottes. Cette quête du moderne est devenu un aspect fondamental des pratiques touristiques chinoises : les sites et les lieux touristiques doivent être bien équipés et facilement accessibles. La grotte Guan, par exemple, satisfait pleinement à ces critères : "Equipped with an automatic light and sound system, the cave now looks more enchanting than ever. In addition, modern facilities such as a railroad car, yacht, and sightseeing elevator provide convenience» (TravelChinaGuide.com, 1998-2010). En ce sens, ce n'est pas seulement la visite de la grotte qui intéresse les touristes, mais aussi ses à-côtés modernes, les possibles activités de magasinage ou les balades en train, bref un certain divertissement.

\section{Les photos sont stéréotypées : l'importance de l'État}

L'ensemble des formes des cavités, comme les collines du fleuve Li, est photographié par les touristes intérieurs et par les touristes internationaux occidentaux. Toutefois, les modalités d'expression du "cliché souvenir» ici encore divergent, à l'instar des pratiques classiques de découverte et de contemplation du paysage. Cela tout d'abord parce que le tourisme intérieur chinois est un phénomène récent, que la différenciation des produits touristiques n'est pas d'actualité à cause du nombre de primo-visiteurs dans chaque site touristique. Comme le souligne Pal Nyíri (2008 : 28), il y a peu de place pour les différences dans le tourisme intérieur chinois : «[D]ans le contexte actuel et récent du développement du tourisme intérieur chinois, quand le plus grand segment du marché se compose toujours des personnes qui n'ont jamais été des touristes, la différentiation des produits n'est pas une priorité.» Le tourisme intérieur demeure profondément standardisé malgré l'apparition de pratiques touristiques plus individuelles. Selon cet argument des pratiques stéréotypées, la logique conformiste se retrouve sur les photos.

Les sites sont en effet largement balisés, marquant des endroits précis où la prise de vue est incontournable. Ces sites correspondent à des vues célèbres, apprises par cour à l'école. Tous les touristes chinois savent par exemple que les plus belles collines à Guilin sont les collines de la trompe de l'éléphant, la colline Fubo et la colline Diecai (en plein centre-ville). La prise de photos stéréotypées constituerait donc l'extension de 
l'éducation et du rôle de l'État, ce que montre encore une fois Nyíri (2008). La volonté de «discipliner» les touristes intérieurs, comme nous l'avons précédemment montrée, est restée une prérogative de l'État par sa stratégie de revaloriser des carnets de voyage (lüyou ji), les descriptions ont été apprises par cœur dans la perspective de former dans la tête des élèves l'idée d'une nation chinoise. Les lieux touristiques chinois sont ainsi des lieux de célébration d'une Chine une et indivisible (Nyíri, 2006; David, 2007; infra).

Dans l'illustration 6, la manière d'entourer une photo d'un cadre avec la date et le lieu n'est encore une fois pas spécifique à la Chine. Cependant, le fait que presque tous les lieux touristiques déploient cette possibilité est révélateur. Dans les sites culturels, comme (et surtout) dans les sites naturels, il y a toujours un photographe pour prendre et retravailler rapidement une photo. Les couleurs sont en effet souvent modifiées dans des tons qui paraissent «kitch» pour les touristes étrangers qui observent la scène.

Comme le souligne Pal Nyíri (2006), il faut y voir le rôle de l'État qui a impulsé et stéréotypé les pratiques touristiques. Cet acteur fondamental n'a cependant fait que reprendre des caractéristiques essentielles du regard que portaient autrefois les lettrés sur la nature. On sait par exemple que les pierres dans les jardins classiques étaient choisies pour les images que suggéraient leurs formes étranges. Les roches devaient donc stimuler l'imagination, car elles forment un contraste avec les courbes des autres éléments naturels ou même des éléments créés par l'homme. L'État est donc un acteur important, mais il n'a fait que réactualiser le regard ancestral que des générations chinoises ont porté sur la nature et les phénomènes naturels.

Une conséquence de cet «endoctrinement» (Nyíri, 2008) est visible sur les sites : l'endroit précis où la photo doit être prise est fléché par de nombreuses indications tel «le meilleur endroit pour une photo", etc. Ces sites sont souvent matérialisés par des chaises où est stipulé le nom du site ainsi que la date. De plus, de chaque côté de la chaise, sont écrites des phrases dont la lecture se fait de haut en bas et de droite à gauche, comme dans les poèmes traditionnels. La structure même des phrases est stéréotypée : chaque phrase est constituée de sept caractères, ici plus précisément de deux proverbes (quatre caractères chacun; chengyu) et de trois caractères précisant le lieu. Cette structure grammaticale, deux énoncés parallèles, est connue en Chine pour faire l'éloge d'un lieu, d'un homme, d'une situation, comme le montre la photo de l'illustration 6, prise dans la grotte de Zhijin (province du Guizhou) devant la forme de la «bru massant sa belle-mère». Ici le cadre n'est pas celui d'une chaise, mais les mêmes éléments (phrases parallèles) se retrouvent sur la photo une fois développée. Deux énoncés de sept caractères chacun se trouvent bien autour de la photo, vantant la beauté du site : à droite, «ce paysage ne se voit qu'au paradis» et à gauche, «dans le monde, où et combien de personnes peuvent le visiter» (Traduction libre de l'auteur : Traduire fidèlement ces deux expressions est difficile, car ce sont des expressions littéraires. Nous ne donnons donc ici qu'une traduction approximative. Le sens est toutefois bien celui transcrit ici.)?

Celui qui a les moyens de venir ici, de s'y faire prendre en photo et de montrer celle-ci aux autres gagnera immanquablement de la «face». Il faut toutefois relever que la pratique

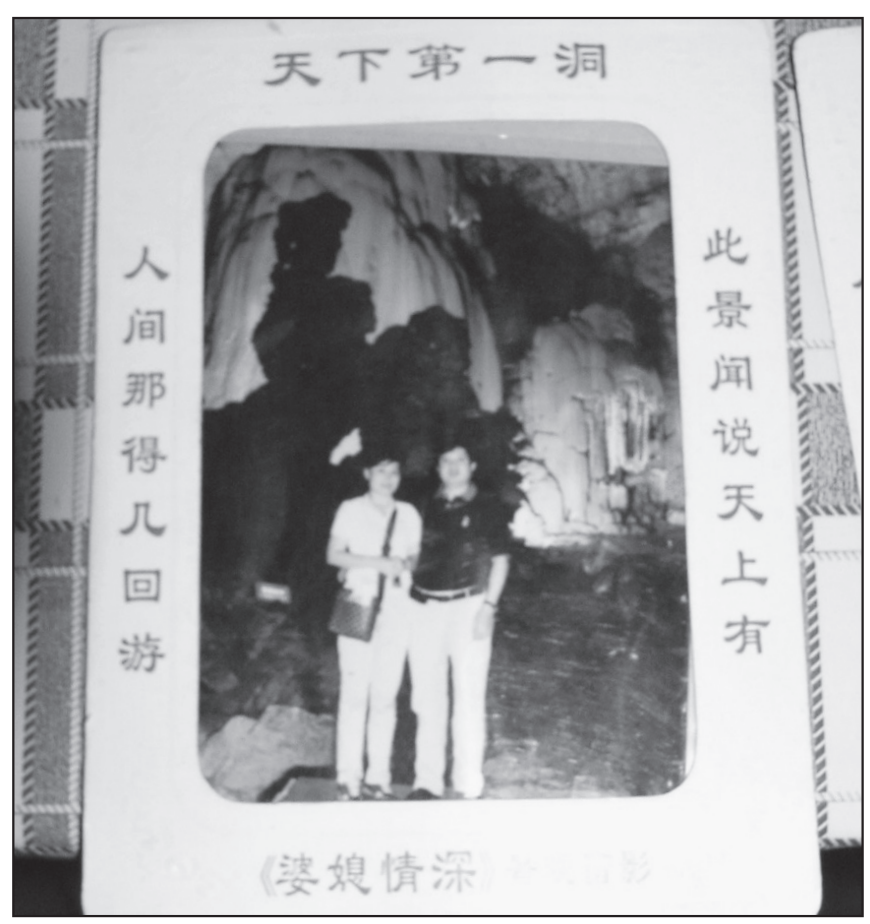

ILLUSTRATION 6 : Benjamin Taunay dans la grotte de Zhijin, au Guizhou (photo : Benjamin Taunay).

de la prise de vue organisée dans les sites touristiques n'est pas propre à la Chine et se retrouve dans de nombreux sites de nombreux pays, ce qui n'empêche pas qu'elle puisse avoir une signification particulière dans la culture chinoise et dans l'approche des géosites. Dans le cas présent, le site est considéré comme la «plus belle grotte sous le ciel» (tian xia di yi dong) expression décidément récurrente : l'utilisation de cette phrase devient tellement galvaudée qu'il devient difficile de déterminer quelle est véritablement la plus belle grotte, la plus belle plage, la plus belle colline, etc. Par exemple, il existe à Guilin deux grottes à qui les aménageurs ont donné le titre de la plus belle grotte du monde... La formule trahit l'implication obsessionnelle de l'État dans la formation des guides, dans le choix des sites touristiques, dans la définition des sites qu'il faut prendre en photo, etc. Il n'est donc pas étonnant que l'ensemble des paysages et des sites connus (les emblèmes; biaozhe $e^{15}$ ) soient payants.

Les touristes intérieurs chinois ne montrent d'ailleurs aucune réticence à payer l'entrée de ces sites nationalement connus et reconnus : ils sont convaincus qu'il faut se faire prendre en photo à un endroit bien précis. Ainsi, devant tous les principaux sites touristiques, une dense haie empêche continuellement de voir gratuitement le spectacle. Ceci est également valable dans les plus petits villages qui «possèdent» un site connu : les habitants des maisons situées à proximité font payer le privilège de prendre la photo depuis chez eux.

\section{Conclusion}

Cet article a permis de mettre en valeur les différentes pratiques des touristes intérieurs chinois dans plusieurs géosites du sud-ouest de la Chine. D’une manière générale, c'est l'aspect 
formel et la signification imagée de la forme qui priment sur la connaissance au sein de ce géotourisme. Les touristes intérieurs observent ainsi les formes des paysages reconnus depuis l'Antiquité chinoise tout en étant attentifs à une certaine recherche de modernité qui n'est pas propre aux pratiques chinoises face à la nature. De plus, la propension de cette population touristique aux analogies rend difficile les interprétations hâtives : comme l'indique un guide à Guilin, "pour voir les formes des collines de Guilin, il y a $30 \%$ d'observation, $60 \%$ d'imagination et $10 \%$ dans mes explications».

L'exemple du sud-ouest de la Chine, espace dominé par un fort relief karstique et visité depuis plusieurs siècles par de nombreux et éminents «karstologues» chinois (tel Xu Xiake), nous montre l'importance du contexte culturel dans les pratiques du géotourisme. Ce n'est pas parce que la Chine accède tardivement au tourisme à l'échelle mondiale qu'elle copie le regard et les pratiques touristiques observés ailleurs, notamment en Occident où est né le tourisme. Le tourisme en Chine ne peut être compris comme un simple transfert des pratiques initiées dans d'autres parties du monde et incorpore nécessairement des éléments propres à la culture chinoise et aux rapports particuliers aux paysages.

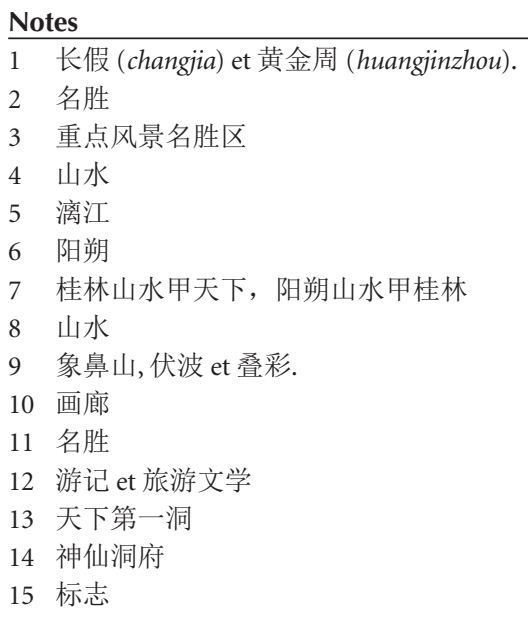

\section{Références}

BERQUE, Augustin (1995) Les raisons du paysage : de la Chine antique aux environnements de synthèse, Paris : Éditions Hazan. 192 p.

BERQUE, Augustin (1997) «La naissance du paysage en Chine», Revue Xoana, $n^{\circ}$ 5, p. 23-29.

CHENG, François (1989) Souffle-Esprit, Paris : Éditions du seuil. 182 p.

CHINESE CULTURAL RELICS TRADE MARKET (2010) «nipic.com», <http://www.nipic.com/show/2/27/f0929478377ea952.html>, consulté le 17 février 2010.

DARS, Jacques (trad.) (1993) Randonnée aux sites sublimes, Paris : Galimard. $392 \mathrm{p}$.

DAVID, Béatrice (2007) «Tourisme et politique : la sacralisation touristique de la nation en Chine», Hérodote, n ${ }^{\circ}$ 125, p. 143-155.

GRANET, Marcel (1999) La pensée chinoise ( $2^{e}$ éd.), Paris : Albin Michel. 569 p. GUO, Chen (2002) Rongbaozhai huapu. Shanshui bufen (album de peintures de Rongbaozhai, volume du paysage), Pékin : Rongbaozhai chubanshe. 42 p.

HU-STERK, Françoise (2004) La Beauté autrement. Introduction à l'esthétique chinoise, Paris : Éditions You-Feng. 225 p.
JULLIEN, François (1993) Éloge de la fadeur, Paris : Éditions LGF-Livre de poche. $158 \mathrm{p}$.

MAIRE, Richard (2004) «Développement du tourisme ethno-karstique dans la province du Guizhou», Chapitre 16 DANS Spéléo-karstologie et environnement en Chine (Guizhou - Yunnan - Liaoning). Voyages en terre chinoise, sous la direction de Jean-Pierre BARBARY, Jean BOTTAZZI, Richard MAIRE, Nathalie VANARA et Shouyue ZHANG, p. 441-456. Karstologia Mémoire $\mathrm{n}^{\circ}$ 9, Lyon : Fédération française de spéléologie.

MING-CHUNG, Ku (2006) The Construction of Self in the Diversified References of Past-ness: Domestic Tourists in China's Heritage Tourism, pré-actes du colloque «Of Asian Origin : rethinking tourism in contemporary Asia» (Singapour: 7 au 9 septembre 2006), <http://www.ari.nus.edu.sg/docs/prog/ pg_tourism.pdf $>$, cité dans NYIRI, Pal (2008) «Between encouragement and control : tourism, modernity and discipline in China», DANS Asia on Tour: Exploring the Rise of Asian Tourism, sous la direction de Tim WINTER, Tommy CHANG et Peggy TEO, p. 153-169. Londres : Routledge.

NATIONAL GEOGRAPHIC SOCIETY (2009) «Charte de géotourisme», NGS, $<$ http://travel.nationalgeographic.com/travel/sustainable/pdf/geotourism_ charter_template.pdf $>$, consulté le 10/11/2010.

NYIRI, Pal (2006) Scenic spots. Chinese tourism, the state, and cultural authority, Seattle et Londres : University of Washington press. $135 \mathrm{p}$.

NYIRI, Pal (2008) «Between encouragement and control: tourism, modernity and discipline in China», DANS Asia on Tour: Exploring the Rise of Asian Tourism, sous la direction de Tim WINTER, Tommy CHANG et Peggy TEO, p. 153-169. Londres : Routledge.

OAKES, Tim (1998) Tourism and modernity in China, New York : Routledge. $272 \mathrm{p}$.

PICKEL, Sylvine (2004) «Représentations et pratiques de la Nature dans les stations atlantiques françaises : une construction sociale évolutive». Thèse de doctorat en géographie, Paris : Université Paris 7-Denis Diderot. 486 p.

SANJUAN, Thierry (2007) Atlas de la Chine, les mutations accélérées, Paris : Éditions Autrement. 79 p.

STOCK, Mathis (2005) «Les sociétés à individus mobiles : vers un nouveau mode d'habiter?», EspacesTemps.net, Textuel, 25 mai.

TAUNAY, Benjamin (2009) «Le tourisme intérieur chinois : approche géographique à partir de provinces du sud-ouest de la Chine». Thèse de géographie, La Rochelle : Université de La Rochelle. 471 p.

THIESSE, A.-M. (1999) La création des identités nationales, Éditions du Seuil : Paris. 385 p.

THIREAU, I. (2006) «Loisir», cité dans SANJUAN, Thierry (2006) Dictionnaire de la Chine contemporaine, Armand Colin : Paris. 303 p.

TRAVEL CHINA GUIDE (1998-2010) «TravelChinaGuide.com : 24/7 Professionnal China Tour Service», TravelChinaGuide.com, $<$ www. TravelChinaGuide.com>, consulté le 8 septembre 2009

URRY, John (1990) The tourist gaze, Leisure and Travel in Contemporary Societies, Londres : Sage Publications. 200 p.

WANG, H.; Z.YANG; L. CHEN; J. YANG et R. LI (2010) «Minority community participation in tourism: a case of Kanas Tuva villages in Xinjiang, China», Tourism Management, vol. 31, $\mathrm{n}^{\circ}$ 6, p. 759-764.

WANG, H. (2004) Guangxi zhuangzu zizhiqu dituji (Atlas de la région autonome Zhuang du Guangxi), xingqiu ditu chubanshe, Beijing, 486 p. 